\title{
The MANIFEST prototyping design study
}

Jonathan S. Lawrence

Sagi Ben-Ami

David M. Brown

Rebecca A. Brown

Scott Case

Steve Chapman

Vladimir Churilov

Matthew Colless

Robert Content

Darren Depoy

lan Evans

Tony Farrell

Michael Goodwin

George Jacoby

Urs Klauser

Kyler Kuehn

Nuria P. F. Lorente

Slavko Mali 


\title{
The MANIFEST Prototyping Design Study
}

Jon S. Lawrence ${ }^{\mathrm{a}}$, Sagi Ben-Ami ${ }^{\mathrm{b}}$, David M. Brown ${ }^{\mathrm{a}}$, Rebecca Brown ${ }^{\mathrm{a}}$, Scott Case ${ }^{\mathrm{a}}$, Steve Chapman ${ }^{\mathrm{a}}$, Vladimir Churilov ${ }^{\mathrm{a}}$, Matthew Colless ${ }^{\mathrm{c}}$, Robert Content ${ }^{\mathrm{a}}$, Darren DePoy ${ }^{\mathrm{d}}$, Ian Evans ${ }^{\mathrm{b}}$, Tony Farrell ${ }^{\mathrm{a}}$, Michael Goodwin ${ }^{\mathrm{a}}$, George Jacoby ${ }^{\mathrm{e}}$, Urs Klauser ${ }^{\mathrm{a}}$, Kyler Kuehn ${ }^{\mathrm{a}}$, Nuria P. F. Lorente ${ }^{\mathrm{a}}$, Slavko Mali ${ }^{\mathrm{a}}$, Jennifer L. Marshall ${ }^{\mathrm{d}}$, Rolf Muller ${ }^{\mathrm{a}}$, Vijay Nichani ${ }^{\mathrm{a}}$, Naveen Pai ${ }^{\mathrm{a}}$, Travis Prochaska $^{\mathrm{d}}$, Will Saunders ${ }^{\mathrm{a}}$, Luke Schmidt ${ }^{\mathrm{d}}$, Keith Shortridge ${ }^{\mathrm{a}}$, Nicholas F. Staszak ${ }^{\mathrm{a}}$, Andrew Szentgyorgyi ${ }^{b}$, Julia Tims ${ }^{\mathrm{a}}$, Minh Vuong ${ }^{\mathrm{a}}$, Lew Waller ${ }^{\mathrm{a}}$, Ross Zhelem ${ }^{\mathrm{a}}$

${ }^{a}$ Australian Astronomical Observatory, North Ryde, NSW 2113, Australia; ${ }^{b}$ Harvard-Smithsonian Center for Astrophysics, Cambridge, MA 02140, USA; ${ }^{c}$ Research School for Astronomy \& Astrophysics, Australia National University, Canberra ACT 2611, Australia; ${ }^{\mathrm{d}}$ Department of Physics and Astronomy, Texas A\&M University, College Station, TX 77843 USA; ' Giant Magellan Telescope/Carnegie Observatories, 813 Santa Barbara Street, Pasadena, CA 91101, USA;

\begin{abstract}
MANIFEST is a facility multi-object fibre system for the Giant Magellan Telescope, which uses 'Starbug' fibre positioning robots. MANIFEST, when coupled to the telescope's planned seeing-limited instruments, GMACS, and G-CLEF, offers access to: larger fields of view; higher multiplex gains; versatile reformatting of the focal plane via IFUs; image-slicers; and in some cases higher spatial and spectral resolution. The Prototyping Design Study phase for MANIFEST, nearing completion, has focused on developing a working prototype of a Starbugs system, called TAIPAN, for the UK Schmidt Telescope, which will conduct a stellar and galaxy survey of the Southern sky. The Prototyping Design Study has also included work on the GMT instrument interfaces. In this paper, we outline the instrument design features of TAIPAN, highlight the modifications that will be necessary for the MANIFEST implementation, and provide an update on the MANIFEST/instrument interfaces.
\end{abstract}

Keywords: fibre positioner, fibre positioning systems, Starbugs, fibre robots, GMT

\section{INTRODUCTION}

MANIFEST is a robotic fibre positioning system designed for the Giant Magellan Telescope. The MANIFEST instrument concept [1-3] consists of hundreds of individual robots, called Starbugs [4-11], which are moved in parallel across the full GMT 20 arcmin field-of-view to very high precision using a closed-loop metrology system. The Starbugs are adhered via vacuum force to a glass field plate located at the GMT focal plane. Each Starbug robot houses a single or multi-fibre IFU payload that transfers light to the entrance slit of the telescope's seeing limited spectrographs: G-CLEF $[12,13]$ and GMACS [14].

The AAO completed a Feasibility Study for the MANIFEST instrument in mid-2011. This was followed by a Research and Development Study in 2012. In 2013, the Prototyping Design Study for MANIFEST was begun. This study, now nearing completion, has focused on developing a working prototype of a Starbugs system called TAIPAN [15] for the UK Schmidt Telescope (UKST), which will be used to conduct a stellar and galaxy survey of the Southern sky.

TAIPAN is a spectroscopic instrument that incorporates 150-300 optical fibres within individual Starbug robots that position across the 6 degree field-of-view of the UKST [16]. In addition to the positioning system prototype for MANIFEST, the TAIPAN project includes a new spectrograph (an all-refractive 2-arm design that delivers a spectral resolution of $\mathrm{R}>2000$ over the wavelength range $370-870 \mathrm{~nm}$ [17]) and an upgrade to the UKST systems (telescope drive motors and encoders, hardware and software control systems, and dome drive and shutter control systems).

In addition to the development of TAIPAN, the MANIFEST Prototyping Design Study has included continued development of the instrument concept to ensure that MANIFEST is optimal and consistent with GMT instrument and telescope designs and interfaces. We are now planning for the Conceptual Design Study for MANIFEST, which is expected to commence in late-2016.

*j1@aao.gov.au; phone +61 2 9372-4853

Ground-based and Airborne Instrumentation for Astronomy VI, edited by Christopher J. Evans, Luc Simard, Hideki Takami Proc. of SPIE Vol. 9908, 990890 · C 2016 SPIE · CCC code: 0277-786X/16/\$18 · doi: 10.1117/12.2232412 


\section{SCIENCE GOALS}

MANIFEST opens new regions of parameter space that complements the stand-alone modes available to the GMACS and G-CLEF instruments. Access to the full telescope field-of-view is provided by distributing fibres across the full telescope focal plane. Higher multiplex gains are obtained because more efficient detector packaging is allowed compared to that achieved using the slit mask of GMACS, and because multiplex can be traded against wavelength coverage for G-CLEF. Spectral resolution and/or wavelength coverage can be increased with no noise penalty because MANIFEST uses image-slicing IFUs coupled to spectrographs that have oversampled detectors. Spatially resolved spectroscopic information can be efficiently obtained using large-format IFUs. These improvements must be balanced against the loss in efficiency that arises with the introduction of the fibre transport system.

The above gains in parameter space lead to a broad range of science objectives that augment the science cases for the GMACS and G-CLEF instruments. Key science goals for MANIFEST are highlighted here:

- Intergalactic medium tomography using Lyman break galaxies: the aim is to reconstruct the 3D small-scale structure of the intergalactic medium at high redshifts by very densely sampling the Ly $\alpha$ forest over large areas of sky [18]. GMT can see the Lya forest in faint (hence dense) Lyman break galaxies at $2<\mathrm{z}<3.5$ in the wavelength range $0.36-0.56 \mu \mathrm{m}$. Relative to GMACS with a standard grating, MANIFEST will double the number of objects and the resolution giving sub-Mpc sampling of the sampling of the IGM along the line of sight.

- Spatially resolved galaxy studies: the SAMI instrument [19] at the AAT uses multi-IFUs for studying spatially resolved galaxies out to $z=0.05-0.1$. It aims for a sample of 3400 galaxies. A future instrument under development at the AAT, Hector [20], will extend this to 100,000 galaxies (at R=4000 over the full optical band). MANIFEST+GMACS could extend this sample out to $\mathrm{z} \sim 0.5$. The broad science program from such a survey include studies of galaxy evolution, kinematic morphological transformation, mass and angular momentum build up, feeding and feedback, and winds and outflows.

- Stellar chemical abundances in Local Group galaxies: this program aims to use chemical abundances [21] to trace the formation history of the Milky Way and nearby Local Group galaxies (the Large Magellanic Clouds, the Small Magellanic Clouds, and $\sim 40$ dwarfs within $\sim 1 \mathrm{Mpc}$ ). MANIFEST combined with G-CLEF provides the only possibility to build up a statistically significant survey. For example, over 30 nights, 2000 stellar spectra at $\mathrm{S} / \mathrm{N} \sim 30$ could be obtained, sampling $\sim 100$ RGB stars in each of 20 Local Group galaxies.

- Archeology of the outer Milky Way disk via chemical tagging: the HERMES multi-object spectrograph at the AAT will use detailed abundances for a million bright stars to chemically tag coeval stellar associations in the inner disk/bulge [22]. MANIFEST+G-CLEF could undertake an extension of this survey to fainter stars, allowing the outer disk and even the inner halo to be probed.

\section{TAIPAN VERSUS MANIFEST}

The TAIPAN instrument has two key aims. This first aim is to act as a proof-of-concept for the Starbugs positioning technology for use on the Giant Magellan Telescope. It is intended that by delivering a working prototype on the UKST, the feasibility of Starbugs technology is demonstrated, thus reducing risk and cost to the MANIFEST instrument for GMT. The second aim for TAIPAN is to deliver a capability to Australian astronomers to carry out a comprehensive spectroscopic galaxy and stellar survey of the Southern Hemisphere using a refurbished UK Schmidt Telescope. These surveys have several scientific objectives in fields of cosmology, galaxy evolution, exoplanets, and stellar astrophysics.

There are many differences between TAIPAN and MANIFEST that will necessitate substantial new design work for the GMT implementation. There are, however, many similarities leading to overlapping requirements and overlapping design solutions. Much of the TAIPAN engineering effort is directly translatable to MANIFEST. This includes hardware designs for electronics control systems, firmware written for low-level Starbug control, and software for Starbug routing, allocation, and close-looped control. Many mechanical designs or subsystem parts can also be replicated. Lessons learnt during instrument commissioning and survey operations are expected to further impact the MANIFEST design.

Table 1 presents a comparison of key parameters for TAIPAN and MANIFEST. In the following sections we provide some discussion of the key instrument subsystems developed for TAIPAN and the work that will be required to develop these subsystems towards MANIFEST in the next project phase: the Conceptual Design Study. 
Table 1. Comparison of key differences between TAIPAN and MANIFEST.

\begin{tabular}{|l|l|l|}
\hline Parameter & TAIPAN & MANIFEST \\
\hline Telescope & UKST & GMT \\
\hline Telescope configuration & Schmidt & Gregorian \\
\hline Telescope diameter & $1.2 \mathrm{~m}$ & $24.5 \mathrm{~m}$ \\
\hline Telescope focal ratio & $\mathrm{f} / 2.5$ & $\mathrm{f} / 8$ \\
\hline Plate scale & $70 " / \mathrm{mm}$ & $1 " / \mathrm{mm}$ \\
\hline Field of view & 6 degrees & 20 arcmins \\
\hline Focal plane diameter & $335 \mathrm{~mm}$ & $1300 \mathrm{~mm}$ \\
\hline Focal plane ROC & $3072 \mathrm{~mm}$ & $3275 \mathrm{~mm}$ \\
\hline Number of Starbugs & $150-300$ & $>200$ \\
\hline Number of fibres & $150-300$ & $>4000$ \\
\hline Starbug pitch & $17 \mathrm{~mm}$ & $50 \mathrm{~mm}$ \\
\hline Positioning accuracy & $5 \mu \mathrm{m}$ & $20 \mu \mathrm{m}$ \\
\hline Payload & Single fibre & IFU \\
\hline Telescope interface & Fixed & Deployable \\
\hline Instrument interface & Single & Multiple \\
\hline
\end{tabular}

\subsection{Starbugs}

Starbugs are the enabling technology for the TAIPAN instrument. A Starbug (see Figure 1) consists of two coaxial piezo-electric tubes that are electronically controlled to flex in a walking action in either of four directions or to rotate on its central axis. The application of appropriate waveforms to the piezo-electric electrodes enables a Starbug to position its payload within $5 \mu \mathrm{m}$ of a target. The primary payload for TAIPAN consists of a single science fibre, which is mounted in a ferrule in the central tube, that allows light to be transferred from the focal surface, through the telescope, and to the TAIPAN spectrograph. The Starbugs are adhered to a glass field plate at the telescope focus via a vacuum that is applied through the annulus between the two tubes. Also fixed within this annulus are three metrology fibres which are illuminated by a single LED that is mounted internal to each Starbug body. For TAIPAN, each Starbug is terminated via a vacuum hose to a single connector that distributes high-voltage drive signals for the piezo electrodes, low voltage signals for the metrology LED, a single optical fibre for the science light, and the vacuum connection. A multi-layered platform, termed the instrument connector plate, distributes the services from the connector via PCBs for the electrical signals, a manifold for the vacuum, and individual furcation tubes for the fibres.

The final design and assembly techniques for the TAIPAN Starbug have been refined to produce a robust, high precision, positioning device. Starbugs have been tested far in excess of their required design life with consistent performance and miniscule failure rate. Since the initial design many reliability concerns have been addressed through experimentation with materials and assembly techniques. All of these aspects are directly transferrable to MANIFEST, which has similar (though somewhat relaxed) requirements on positioning accuracy.

For the MANIFEST implementation, a number of design changes will be required. The largest change is to increase the complexity of the payload. While a single fibre mounted in a ferrule is used in TAIPAN, for MAIFEST each Starbug requires an image slicing IFU that comprises a fibre bundle (that may have 7 to 61 fibres) mounted in a fibre array fixed to an air-spaced microlens array pair. Some development of a similar concept has already occurred as part of the AAO Ultimate [23] project (see Figure 2), though this must be further explored in the MANIFEST CDS. The multi-fibre payload also implies a modification of the connector system. Design options to be explored include separate multipole fibre connectors and separate multipole electrical connectors, or fibre feedthroughs without connectors and separate multipole electrical connectors. It is possible that these changes to the Starbug format may require an increase in the 
Starbug size due to the complexity of the payload (i.e., a larger space envelop is needed) or the weight of the payload (to increase the vacuum force).

One aspect of the Starbugs connectorisation system that reduces in complexity for MANIFEST relative to TAIPAN arises due to the density of connections. For TAIPAN, the nominal pitch (i.e., the separation of Starbugs on the field plate in their home position, which is approximately equivalent to the spacing of connectors on the connector plate) is $17 \mathrm{~mm}$. It has proven challenging to fit all required elements within this footprint. For MANIEST, the requirement is relaxed $>50 \mathrm{~mm}$ pitch because of the larger plate size, allowing significantly more spacing to distribute services.
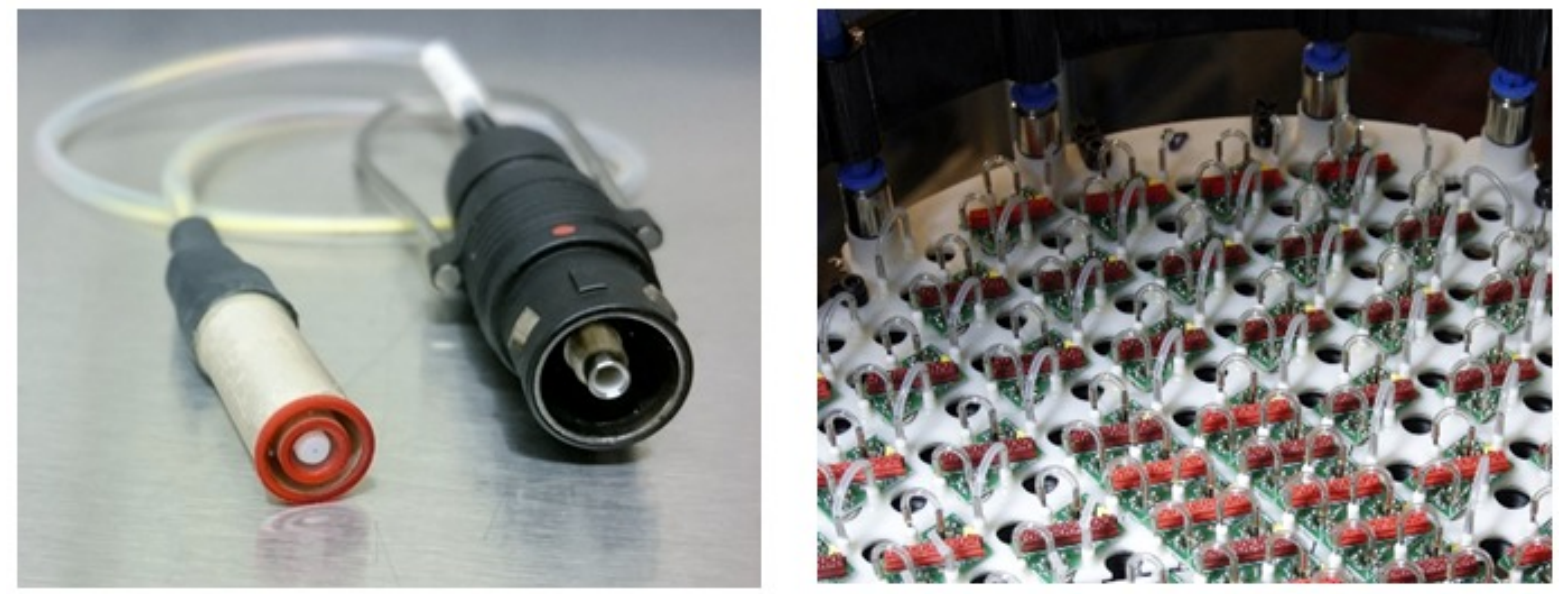

Figure 1. Individual Starbug for TAIPAN (left) and TAIPAN connector plate (right).

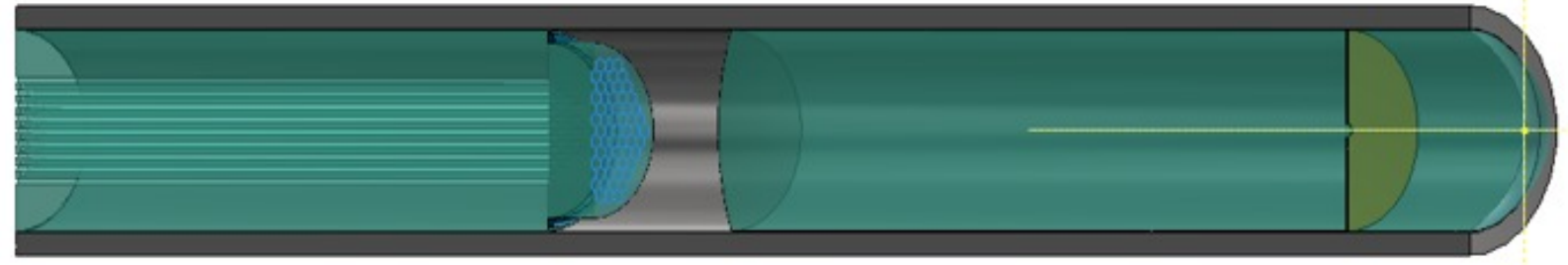

Figure 2. Concept for miniature fibre IFU that fits within the footprint of a Starbug.

\subsection{Positioner assembly}

The TAIPAN positioner (shown in Figure 3 and Figure 4) locates the Starbugs on the glass field plate at the telescope's focal plane and provide all services necessary for the Starbugs to operate. The positioner design is comprised of two main structural assemblies. The primary instrument assembly supports the glass field plate, glass field plate tip-tilt focus drive, Starbugs, bug catcher, connector plate, and provides an interface to the utility spider assembly. The secondary structural assembly is the utility spider whose purpose is to fix the primary instrument assembly to the telescope and to route all required services to the periphery of the telescope while minimizing cross sectional area.

A key function of the positioner is to control the deployment and adherence of the Starbugs onto the glass field plate. This process involves pointing the Schmidt telescope at zenith such that the Starbugs are held from the connector plate vertically above the glass field plate. The connector plate is then driven downwards when the vacuum system is switched on, so the Starbugs make contact with the field plate. To achieve $100 \%$ adherence via contact that is perpendicular to the field plate, a guide (called the bug-catcher) is moved towards the field plate in parallel with the connector plate.

For MANIFEST, the functions of the positioner subsystem are similar, i.e., to locate and position the Starbugs and field plate and to distribute Starbug services. There are two key differences required for the MANIFEST concept.

Firstly, the primary instrument assembly must be increased in size by a factor of $\sim 4$. The full focal plane is $335 \mathrm{~mm}$ for TAIPAN and $1300 \mathrm{~mm}$ for GMT. While many concepts and components can be re-used, design work will be required to account for this scaling in size and mass. 


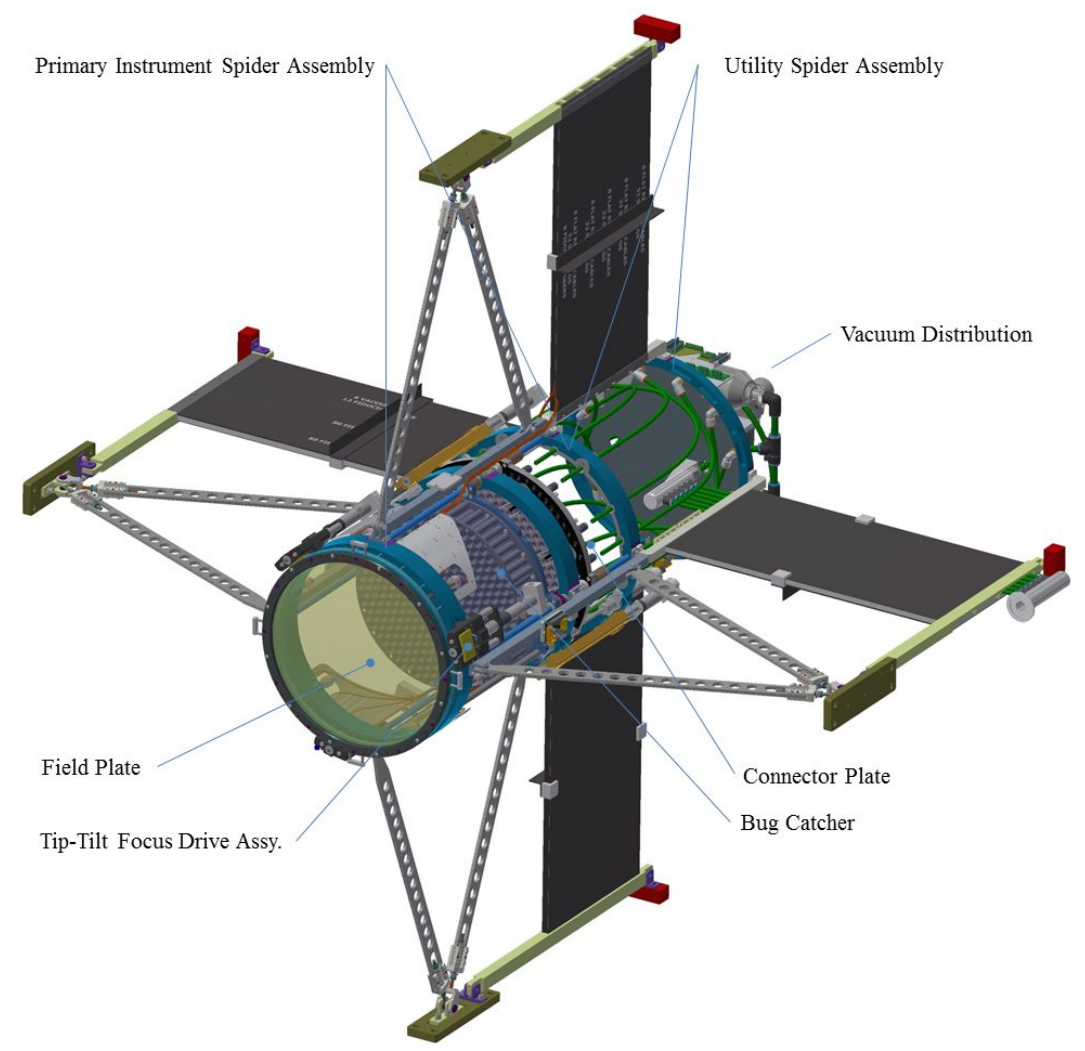

Figure 3. Design for positioner structure for TAIPAN.
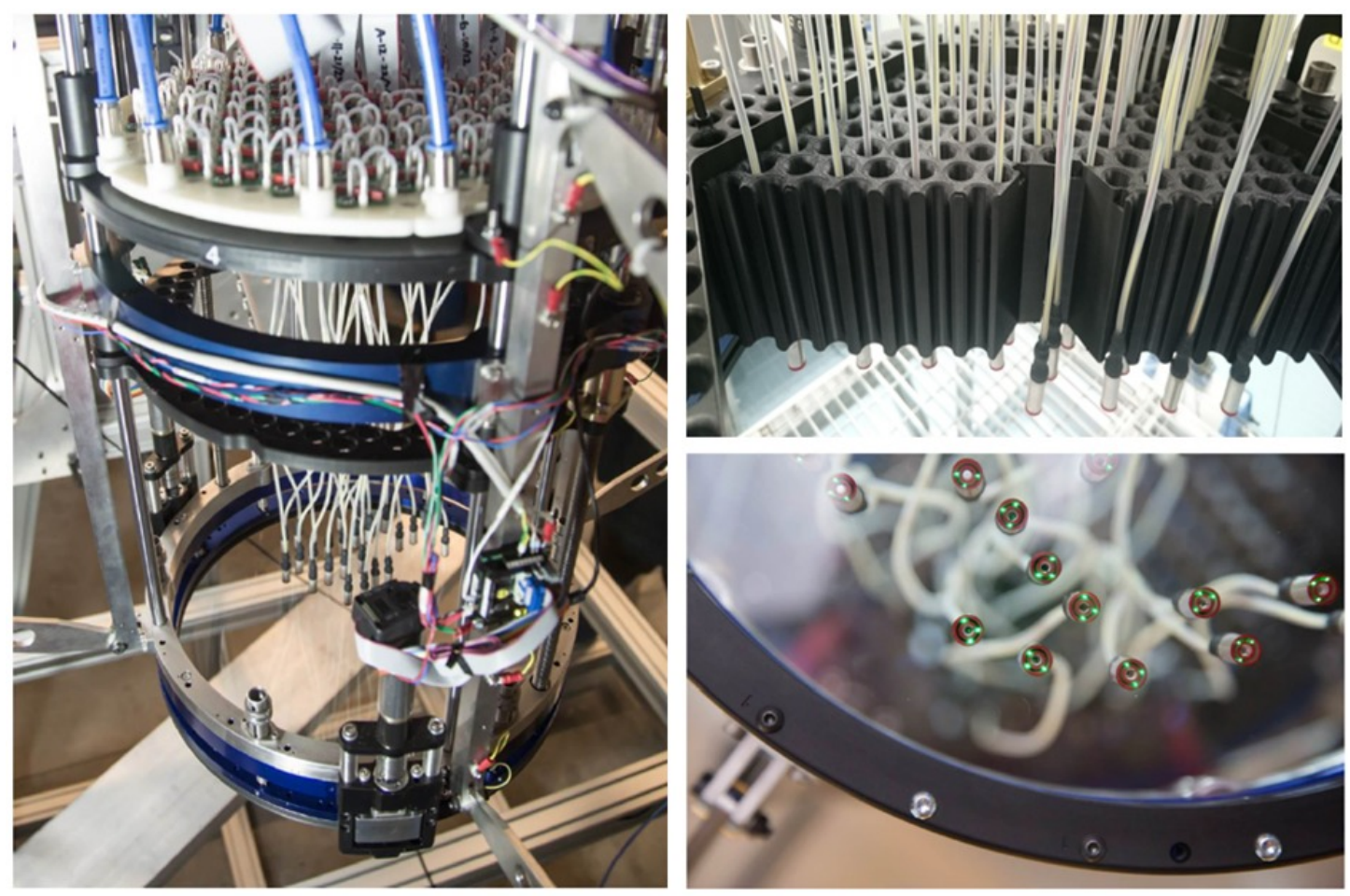

Figure 4. Pictures of TAIPAN positioner showing primary support structure (left), section of bug-catcher (top-right), and view through the glass field plate of Starbugs with lit metrology fibres (bottom-right). 
Secondly, the spider assembly is not required for MANIFEST. Rather, an assembly is required to mount and position MANIFEST in one of the GMT Gregorian Instrument Rotator (GIR) bays. These bays allow the instrument to be deployed to the centre of the telescope focal plane for operation and moved to a stow location when not in use. The design developed during the MANIFEST feasibility study used half an instrument bay for storage with elevation and translation movements required for operation (see Figure 5). Early in the project, however, the mechanism for Starbug adherence to the glass field plate was magnetic coupling, in which the Starbugs were permanently fixed to the field plate. With vacuum coupling, a mechanism for deployment of the Starbugs onto the field plate is now required. The deployment mechanism used for TAIPAN is likely to be suitable for MANIFEST, however, it requires the connector plate to be above the field plate. This solution will require a tumbling mechanism for the primary instrument assembly. Alternative mechanisms for Starbug adherence to the field plate from underneath will be explored during the MANIFEST CDP.

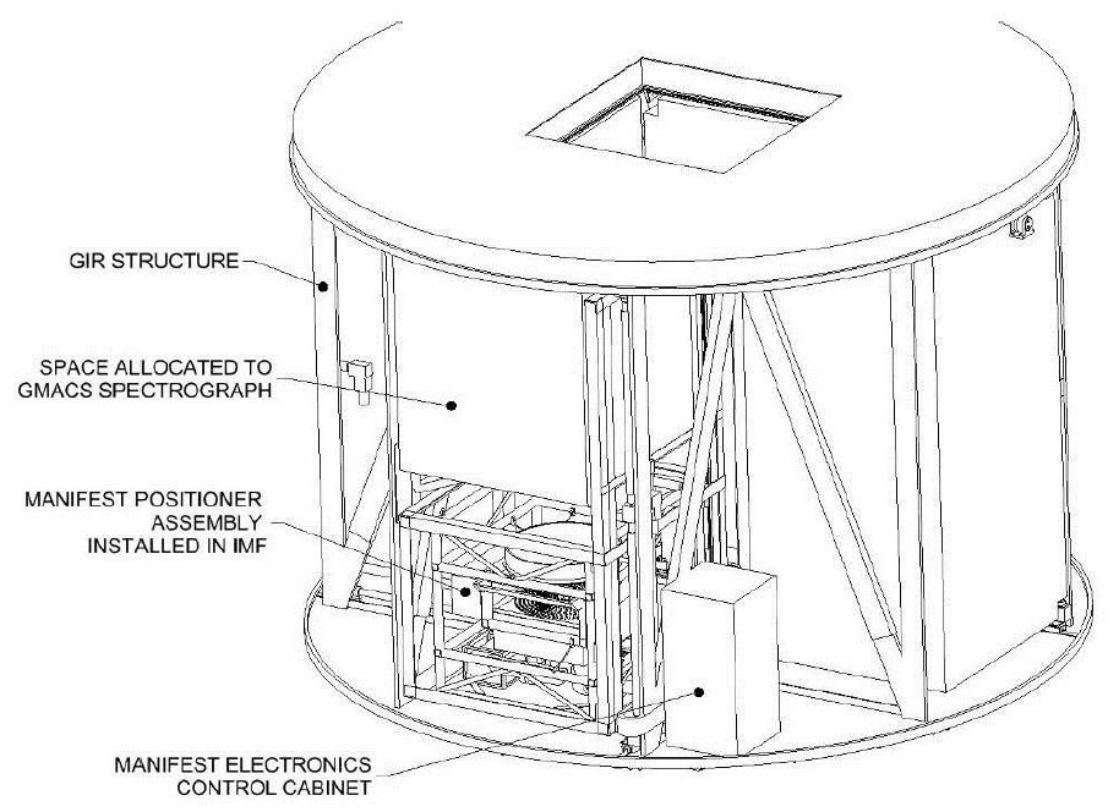

Figure 5. Design for MANIFEST/GMT interface developed during feasibility study.

\subsection{Field plate}

The TAIPAN field plate provides a support interface for the Starbug robots and allows light injection into the science fibre payload carried by the Starbugs. The design of the field plate flowed from the optics of the UKST, an aspheric corrector lens and spherical primary mirror. The field plate is a zero power N-BK7, $5 \mathrm{~mm}$ thick, meniscus lens with a radius of curvature of $3072 \mathrm{~mm}$. Its concave surface is coincident with the telescope focal surface. The field plate has a $335 \mathrm{~mm}$ clear aperture to cover a 6 degree field of view and a total outer diameter of $350 \mathrm{~mm}$ to allow for a mounting bezel. The field plate is mounted in a 410 stainless steel mount and is radially athermalized with an RTV bond. It is axially retained with an aluminum cover and compliant o-ring. Holes on the perimeter of the mounting bezel allow visualisation of 16 back illuminated metrology fiducials. The optic is AR coated on the convex side with a high transmittance coating giving $0.55 \%$ average reflectance over $360-1000 \mathrm{~nm}$. The concave side is coated with a more durable coating giving $1.1 \%$ over the same range.

The MANIFEST field plate nominal design, developed during the Feasibility Study, was for a $3 \mathrm{~mm}$ thick N-BK7 meniscus of diameter $1300 \mathrm{~mm}$ (i.e., to cover the full 20 arcmin field-of-view of GMT) and radius of curvature $3275 \mathrm{~mm}$ (to match the telescope focal plane curvature). Because the initial Starbug design used magnets on either side of the field plate for adhesion the plate thickness had to be $<5 \mathrm{~mm}$ to achieve sufficient magnetic attraction and holding force. It proved challenging to identify glass vendors who could fabricate such a high aspect ratio optic without folding the substantial associated risks into the cost estimate. 
With Starbug vacuum adhesion, there is no longer a constraint on the field plate thickness. However, the field plate design is dependent on the telescope corrector optics, which are not yet finalised. The baseline GMT corrector system design [24] uses an Amici ADC with two counter-rotation prismatic glass doublets for correction of the full telescope field of view. Alternatives have been proposed that also cover the full field of view, including one using a compensating lateral ADC [25], or a single laterally moving doublet [e.g., 26]. An option being considered to reduce costs has a limited field-of-view WFC/ADC that is designed specifically to improve image quality over only the central $\sim 8$ arcmin i.e., covering the GMACS slit plate size. A further cost reduction option has no WFC/ADC at all and accepts reduced image quality and a more limited field. The design for the MANIFEST glass field plate will be considered in the CDS phase as the design for the telescope corrector progresses.

\subsection{Fibre cable}

The fibre cable block diagram for TAIPAN is shown in Figure 6. The cable interfaces the positioner and Starbug via a customized connector at the rear of the connector plate. From the connector the fibre cable carries individual fibres in $2 \mathrm{~mm}$ furcation tubes to consolidation manifolds located near the exit hatch of the telescope. Each manifold receives 25 of the $2 \mathrm{~mm}$ furcation tubes and consolidates them to one $3.8 \mathrm{~mm}$ furcation tube in order to reduce the cable size. The $3.8 \mathrm{~mm}$ furcation tubes, now carrying 25 fibres each, are further protected in a $25 \mathrm{~mm}$ internal diameter, galvanized steel, polyurethane coated, industrial flexible conduit, which protects the fibres and furcation tubes as they are routed to the spectrograph. The fibre conduit exits the telescope entry hatch, is passed through the telescope equatorial bearing, through a hole in the telescope pier and enters the spectrograph room located beneath the telescope. The fibre cable is terminated in a slit assembly that combines the fibres from sets of three furcation tubes into 75 fibre silica V-groove arrays mounted against the spectrograph field lens.

The MANIFEST fibre cable will substantially differ from the TAIPAN fibre cable as its termination is determined by the instruments it is feeding (see Section 4), and the route is determined by the GMT telescope architecture. However, many design features will be similar as we will follow the standard AAO methodology for fibre packing, protection, and routing. This methodology has been established based on many previous AAO fibre cable builds [27,28] that have satisfied key requirements for minimizing focal ratio degradation.

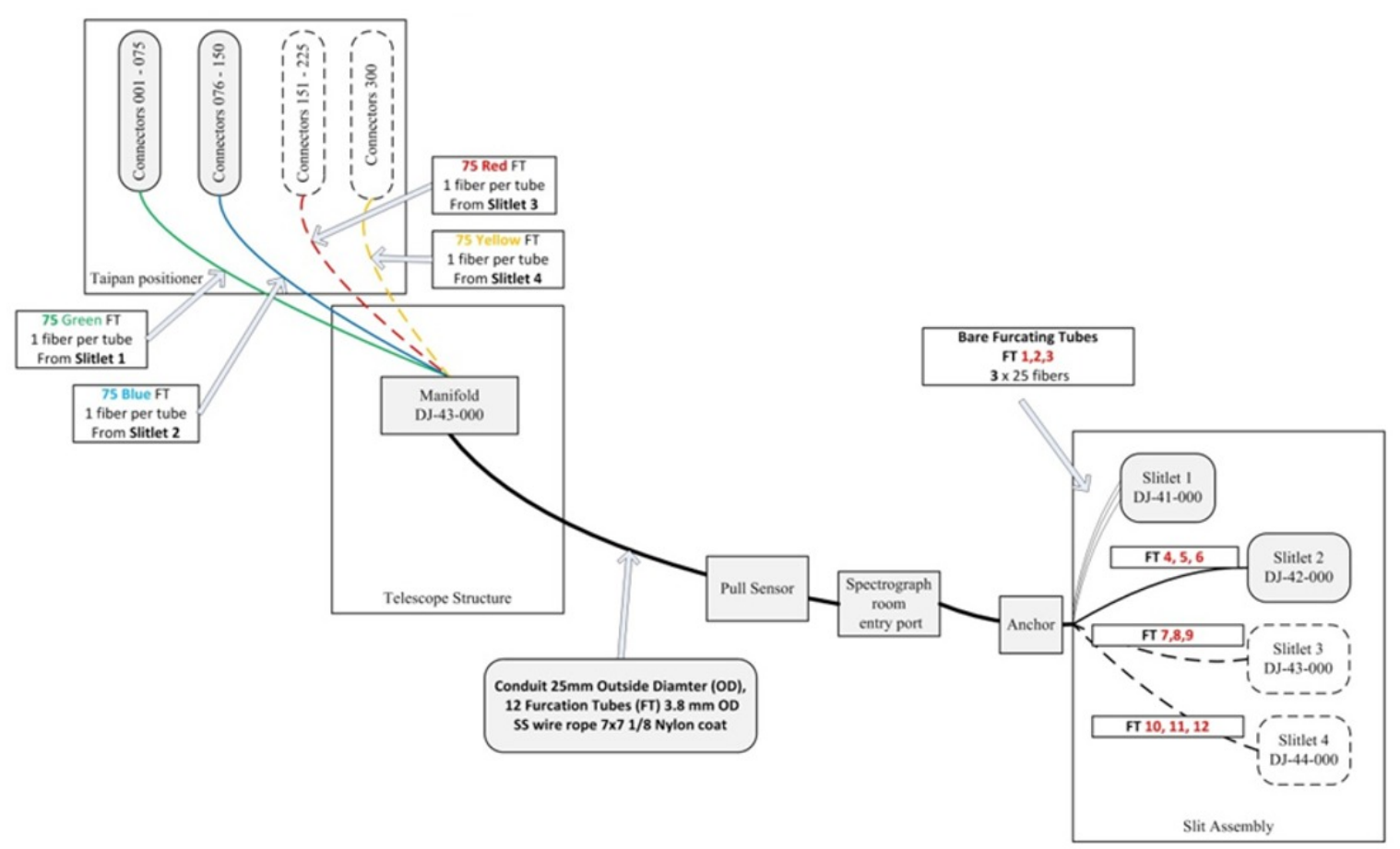

Figure 6. Fibre cable block diagram for TAIPAN. 


\subsection{Metrology}

The metrology system requirement for TAIPAN is to provide a positional measurement for closed loop control of all 150-300 Starbugs to better than $5 \mu \mathrm{m}$ over the full field plate diameter of $0.33 \mathrm{~m}$. The metrology system hardware (see Figure 7) consists of a 29 megapixel monochrome CCD machine vision camera (Prosilica GT6600) with a $250 \mathrm{~mm}$ fixed-focus low-field-distortion $(\sim 0.1 \%)$ compound lens (Hasselblad Tele-Tessar) and a long-focus adjustment lens. The metrology camera is located in the central hole of the primary mirror, mounted directly to the mirror cell, and images the telescope focal plane $\sim 3.5 \mathrm{~m}$ distance. With a focal plate pixel scale of $\sim 77 \mu \mathrm{m}$ per pixel, the required accuracy is then $\sim 1 / 20$ pixel, similar to that achieved by other systems (e.g., 1/30 pixel for DESI [29]). Each Starbug has three LEDilluminated $200 \mu \mathrm{m}$ metrology fibers for positional and directional measurements, providing $\sim 2.5$ pixel sampling for optimal centroiding. The field plate has a set of 16 illuminated fiducials located on its perimeter. These fiducials provide a reference mechanism for the camera distortion model as well as to compensate for any minor movements (from the camera mount or optics) between telescope field pointings.

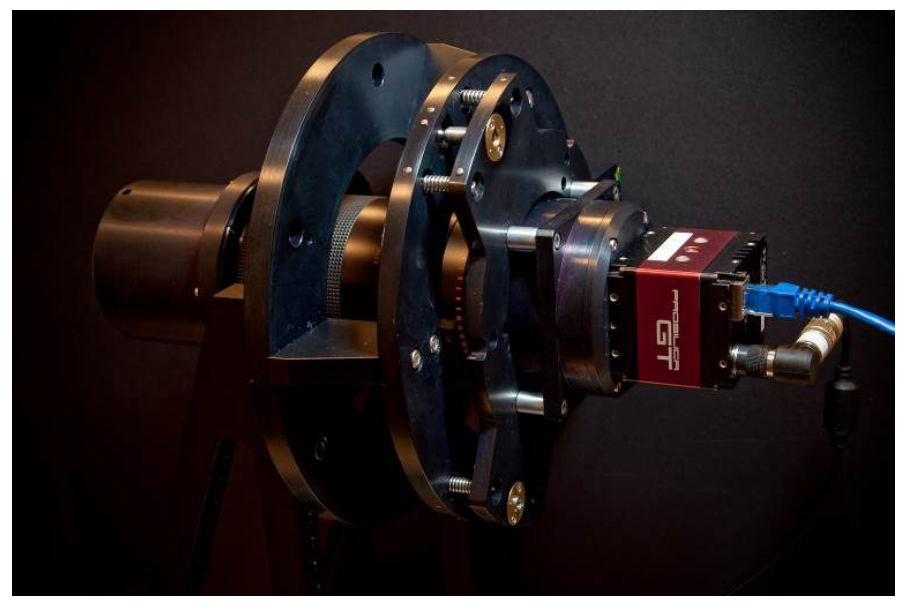

Figure 7. Metrology system camera assembly for TAIPAN.

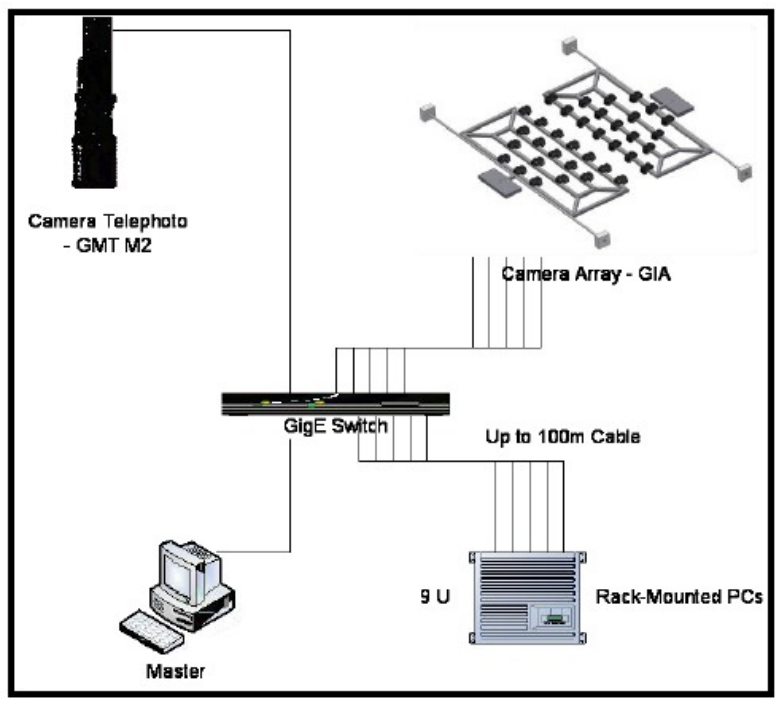

Figure 8. Proposed architecture for the MANIFEST metrology system.

The MANIFEST metrology system has a similar driving requirement (it needs to achieve $20 \mu \mathrm{m}$ precision over $1.3 \mathrm{~m}$ ) to TAIPAN $(5 \mu \mathrm{m}$ over $0.33 \mathrm{~m})$, and will have many common features. The architecture proposed for MANIFEST is shown in Figure 8. It consists of a telephoto camera lens with large format CCD viewing the glass field plate metrology fibres. It is yet to be determined exactly where on the GMT the camera is mounted; options include on a deployable arm below the M2, fixed between the M2 segments in the inner or outer gaps, or along the secondary truss. The light path to 
the metrology camera must be analyzed trading off viewing the field plate directly through the corrector or off the primary and secondary mirrors and then through the corrector. In addition to the on-sky metrology camera a second system is envisaged for engineering purposes, so that system tests can be run while the instrument is in the stowed location. This camera system will need an array of cameras mounted within the instrument frame, and will need stitching algorithms to combine images.

Open loop positioning can be monitored using the metrology camera system. For TAIPAN there is no requirement for open-loop operation as the positioner feeds a single instrument, the science objectives for the instrument call for a maximum exposure time of 15 minutes, and any positioning errors due to atmospheric refraction induced field rotation over this timeframe are negligible because the UKST has the capability of adjusting the telescope polar axis angle.

For MANIFEST on the GMT, there may be strong science drivers for multiple instruments to operate at the same time, and there are no assumptions yet made about maximum instrument exposure time. It will thus be important to understand the open loop precision, i.e., so that Starbugs could move during an exposure to follow atmospheric refraction or to allow independent exposure times for different co-observing instruments. Laboratory tests for TAIPAN have given some indication of achievable precision but ultimately on-sky measurements will be needed.

\subsection{Electronics}

For TAIPAN, the Starbugs are driven by custom AAO designed control electronics installed in $4 \mathrm{U}$ chassis. Each chassis is an independent unit controlling up to 80 Starbugs. Each chassis comprises: a power supply module, which converts $230 \mathrm{VAC}$ to $24 \mathrm{VDC}$ at $17 \mathrm{~A}, 400 \mathrm{~W}$; a high-voltage DC-DC module, which converts $24 \mathrm{VDC}$ to $\pm 200 \mathrm{VDC}, 150 \mathrm{~W}$ for the high voltage piezo actuator drive; a network and waveform module, which provides a $100 \mathrm{Mbps}$ TCP/IP Ethernet to CAN bus communications interface and generates low-voltage Starbug control waveforms; a linear power amplifier module, which converts the low voltage Starbug control waveforms to high-voltage (up to $\pm 200 \mathrm{~V}$ ) piezo actuator drive waveforms; 10 solid-state relay modules, which each provide 224 high voltage switches to apply a matrix of drive waveforms onto the piezo actuator electrodes of 8 Starbugs; and a backplane module, which provides the bussed architecture for $24 \mathrm{~V}$ power, CAN bus communication, timing signals, $\mathrm{HV}$ enable, slot addresses and error signals.

The Starbugs control electronics comprises a total of 15 ARM7 32-bit processors per chassis running at $168 \mathrm{MHz}$. Even though each module is different, it shares the same processor block, with a common image of the firmware (written in $\mathrm{C} / \mathrm{C}++$ ). Inter processor communication is via $\mathrm{CAN}$ bus and timing signals. The timing signals are generated by the waveform module in synchronisation with the waveform signals. New firmware can be uploaded to the external flash memory on each processor using the network module. The high level software sends commands to the network interface such as: power up, power down, setup waveform, start, stop, move bugs, rotate bugs, set bug light intensity and read system status. The system status reports the status and state of each processor module to the high level software.

The MANIFEST Starbugs control electronics will use the same architecture and most of the same electronics boards, processor hardware, and low level firmware as for TAIPAN. One key change planned will be to replace the linear power amplifier module with a switching power amplifier module that will increase the current drive capability so that 160 Starbugs can be controlled with a single chassis.

\subsection{Software}

The TAIPAN positioner software [30] is responsible for closed-loop control and monitoring of the science and auxiliary Starbugs on the field plate. It determines the current location of each Starbug, assigns its next target position, calculates a valid trajectory and sends the appropriate control commands via the electronics firmware layer to move it to its new target. Additionally the software system collects and displays monitor information to ascertain the health of the instrument. Finally, the software also stores monitoring data for the long-term characterisation and evaluation of the instrument's performance. The software is written in $\mathrm{C}++$, using DRAMA for its middleware layer [31], and MongoDB for its database needs.

For MANIFEST, many aspects of the TAIPAN software can be re-used. The positioner module is a stand-alone system with sub-modules for routing, metrology, motion control, calibration, and monitoring that is fully translatable to MANIFEST. The spider mechanism module, which includes the bug-catcher, field plate focus and tip-tilt, and the vacuum system, is partly translatable. Some aspects of the acquisition and guide system and telescope interface systems may also be re-usable. The main software task MANIFEST will be to rewrite the middleware layer in whatever form appropriate for GMT, the instrument interface software, and control systems for any new features. 


\subsection{A\&G}

The acquisition and guiding system for TAIPAN uses nine Starbug-mounted guide probes that are positioned evenly over the 6 degree field plate. Each guide probe uses a $0.5 \mathrm{~mm}$ coherent polymer fibre bundle having approximately 7000 cores with an equivalent field of view of $33^{\prime \prime}$ and a resolution of $\sim 0.4^{\prime \prime}$. The back-end of all nine guide probe bundles are grouped together and re-imaged onto a single camera (GT2450 CCD with telecentric lens) that interfaces to the instrument and telescope control systems.

The guide probe system provides a set of key functions and abilities. It provides: an acquisition camera; a real-time pointing solution for telescope guiding; a robust reference frame (in-field plate fiducials) to improve the positioning accuracy of the science fibers based on an astrometric solution for a given telescope field pointing; a focal plane imager to map out the telescope distortions during commissioning and maintenance operations; the ability to precisely determine the optimal field plate alignment, orientation, and focus for best image quality; and the ability to monitor diagnostics such as seeing, image quality and sky extinction over the field for improved science results.

The GMT will have a laser metrology system to maintain alignment over telescope observing angle for various elements within the optical train. It is possible that this metrology system could be extended to the glass field plate for MANIFEST. However, it will still be necessary to have some form of monitoring system that provides an on-sky reference frame. We envisage the same guide probe system being used on MANIFEST as for TAIPAN, with $\sim 10$ Starbug-mounted guide bundles distributed across the field of view. It is yet to be determined how the data is fed back into the GMT telescope control system. One solution would use the telescope AGWS (Acquisition, Guide, and Wavefront Sensors) system for real time control and the MANIFEST system for slow drift and flexure compensation.

\section{INSTRUMENT INTERFACES}

\subsection{G-CLEF}

The GMT-CfA, Carnegie, Catolica, Chicago Large Earth Finder (G-CLEF) is a fibre fed, optical echelle spectrograph that has been selected as one of the first light instruments on the GMT. G-CLEF [12-13] has been designed to be a general-purpose echelle spectrograph with precision radial velocity capability. The spectrograph optical design (see Figure 9) is an asymmetric, two-arm, white pupil design, with a nominal beam size of $\sim 300 \mathrm{~mm}$ at the primary disperser, and $\sim 200 \mathrm{~mm}$ for the cross-dispersers. The spectrograph has no moving parts and delivers a continuous wavelength coverage of 350-1000 nm. The stand-alone spectrograph is fed with four sets of fibres to enable four observing modes: high-throughput $(\mathrm{R} \sim 20 \mathrm{k})$, precision-abundance $(\mathrm{R} \sim 40 \mathrm{k})$, precision radial velocity (at $\mathrm{R} \sim 100 \mathrm{k}$ ), and non-scrambled precision radial velocity (at $\mathrm{R} \sim 100 \mathrm{k}$ ). The G-CLEF front end consists of a relay system with a pair of triplets and an ADC mounted at the telescope GIR.

The addition of MANIFEST provides an important fifth mode for G-CLEF to enable multi-object spectroscopy. The interface between MANIFEST and G-CLEF is under development. The current concept (see Figure 10) consists of a single row of 40 fibres mounted beside the G-CLEF fibres inside the instrument cryostat. Each fibre is fed by a singlefibre Starbug unit from the MANIFEST glass field plate. With all but the central 4 probes output masked on the spectrograph entrance slit, a multi-order spectra is obtained for 4 objects at once over the $370-1000 \mathrm{~nm}$ range. With all 40 probes and a wedged Fabry-Perot filter, a series of $\sim 7$ well-separated chunks of the spectrum is obtained, tunable in the sense that the whole series can be shifted up and down in wavelength. Any desired echelle order can be selected, along with sections of other orders. This gives a higher multiplex, but at the expense of spectral coverage.

The G-CLEF MANIFEST slit consists of a linear fibre array that is bonded to the first planar face of an air-spaced microlens array pair. The micro-optics are used to magnify the $\sim \mathrm{f} / 3$ fibre beam to the spectrograph $\mathrm{f} / 8$ and also transfers the fibre far field to the pseudo-slit plane. The fibres will exit the G-GLEF cryostat through a vacuum flange with fibre feedthroughs. A mechanism external to the cryostat will allow light from unused fibres to be blocked in low-multiplex MANIFEST mode or for G-CLEF stand-alone operation. A mechanized filter system external to the cryostat will be used to prevent overlapping orders in the MANIFEST high multiplex mode. The fibre cable will join the MANIFEST connector plate via a route through the telescope structure with a cable wrap at the GIR interface. It is anticipated that each fibre will be a bonded into a single-fibre Starbug unit providing $\sim 0.7$ " diameter on-sky sampling, and a spectral resolution similar to the G-CLEF precision-abundance mode. The details for the filter and mask system, the optical configuration for the slit micro-optics, and the potential for including a second row of 40 fibres to further increase the multiplex advantage, will all be explored further in the MANIFEST CDS phase. 


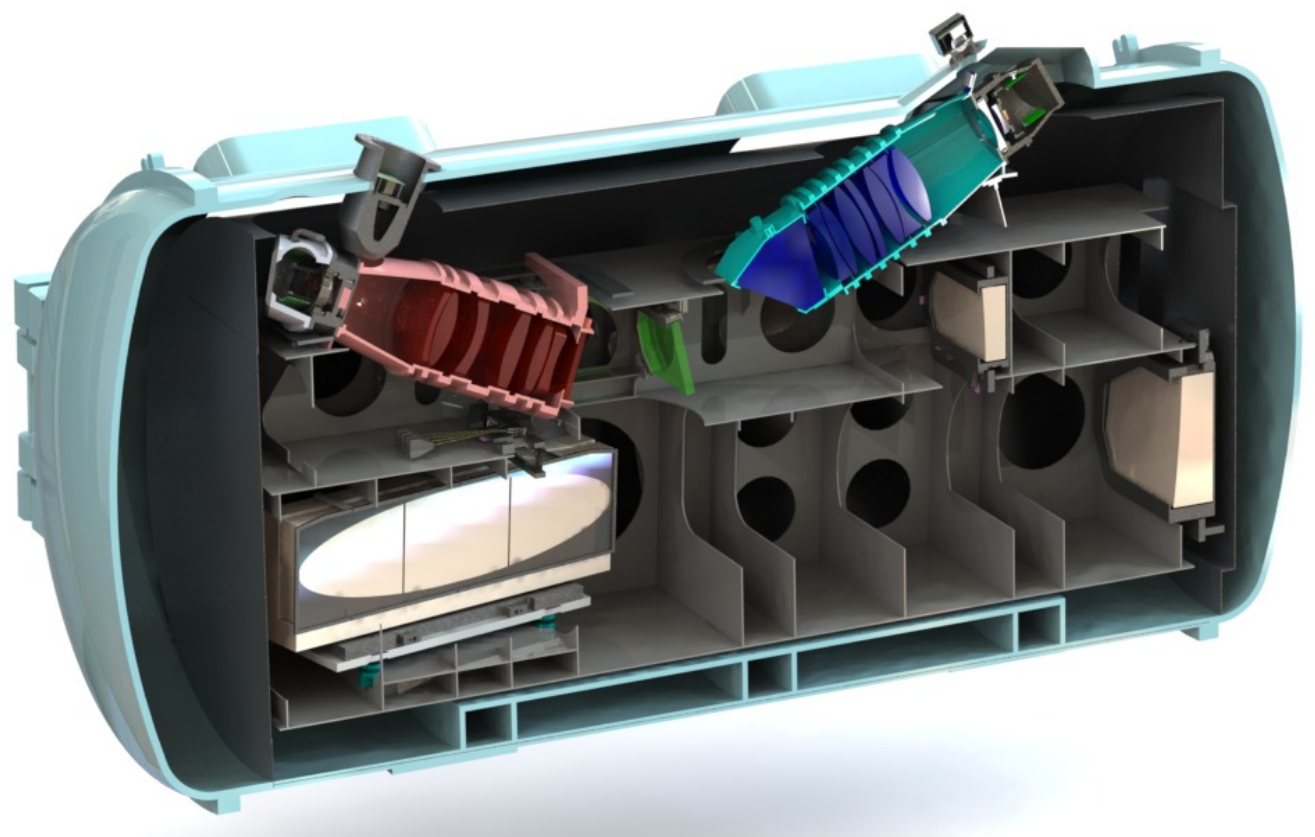

Figure 9. Cut-away model of G-CLEF cryostat showing the two camera arms (red and blue), dichroic (green), collimating mirrors and echelle grating ( $\tan )$ and slit assembly (directly under the red camera arm).
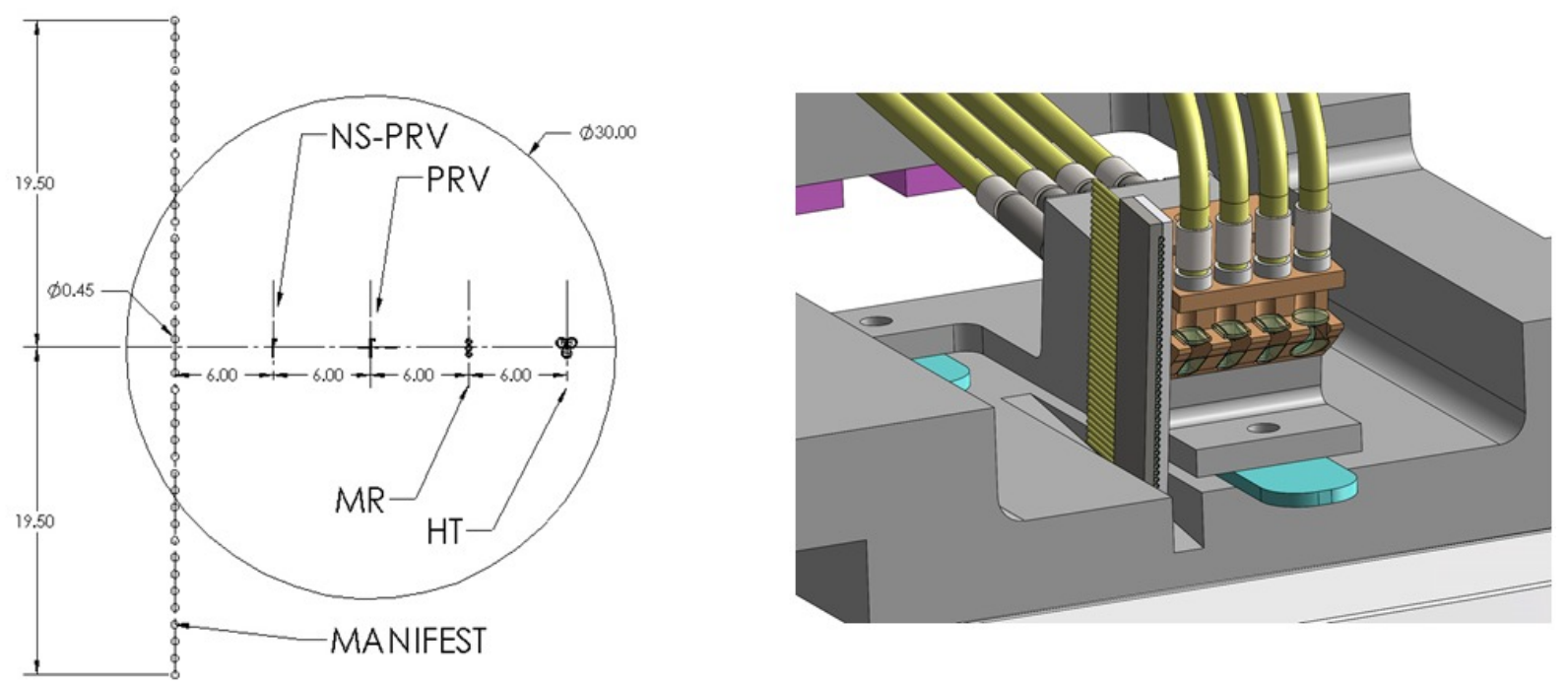

Figure 10. Concept for the G-CLEF pseudo slit layout (left), and opto-mechanical assembly (right).

\subsection{GMACS}

GMACS is a multi-object slit-mask spectrograph that covers a relatively wide field (at least 7.5 arcmins) with a wide wavelength coverage [14]. A dichroic mirror feeds two cameras optimized over single octave bandpasses. The full instrument will deliver moderate resolution $(\mathrm{R}=1000-6000)$ spectroscopy from the near UV out to $\sim 1000 \mathrm{~nm}$. The instrument (see Figure 11) is mounted within a single bay of the GMT GIR. In the top section of the instrument frame is the slit mask holder which sits at the telescope focal plane when the instrument is deployed. Twin cassettes, mounted either side of the focal plane, position and automatically exchange slit masks into the holder. Two collimating lens groups deliver light via a dichroic to two separate camera arms containing volume phase holographic gratings. The gratings and camera barrels for each arm are articulating, and gratings can be swapped in and out to provide variable spectral resolution. 


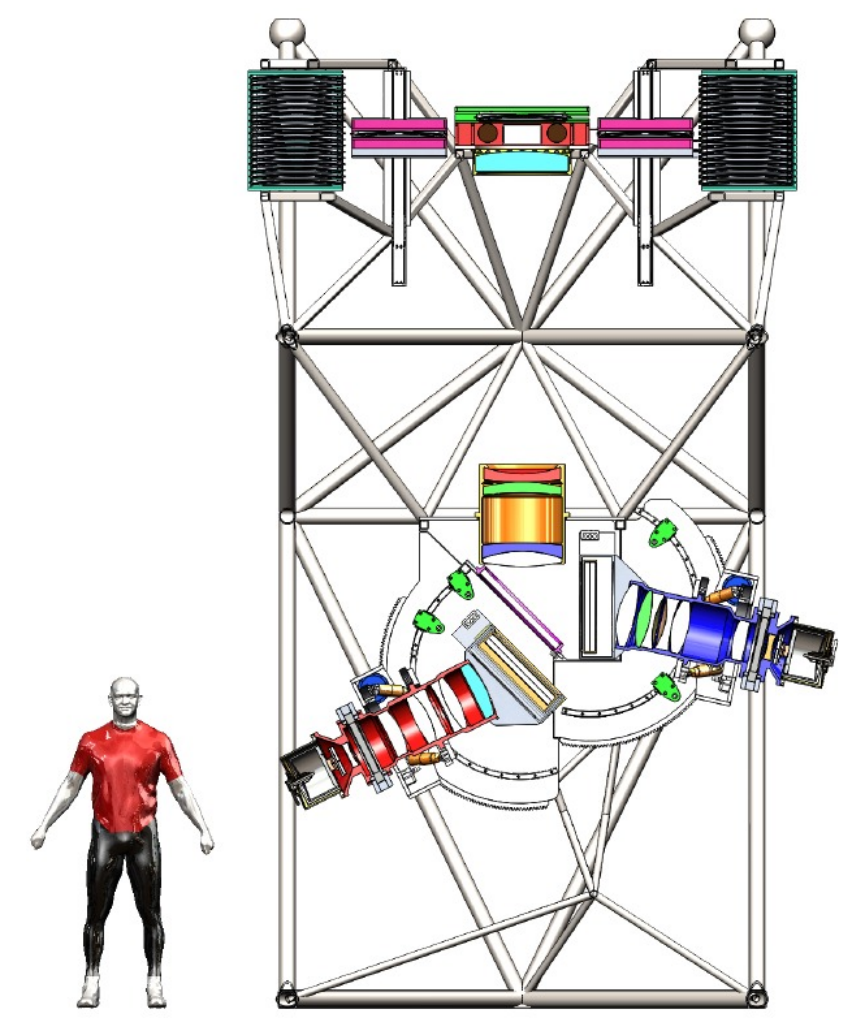

Figure 11. Concept model for the GMACS spectrograph, showing slit-exchange unit feeding collimator optics with twodeployable camera arms.

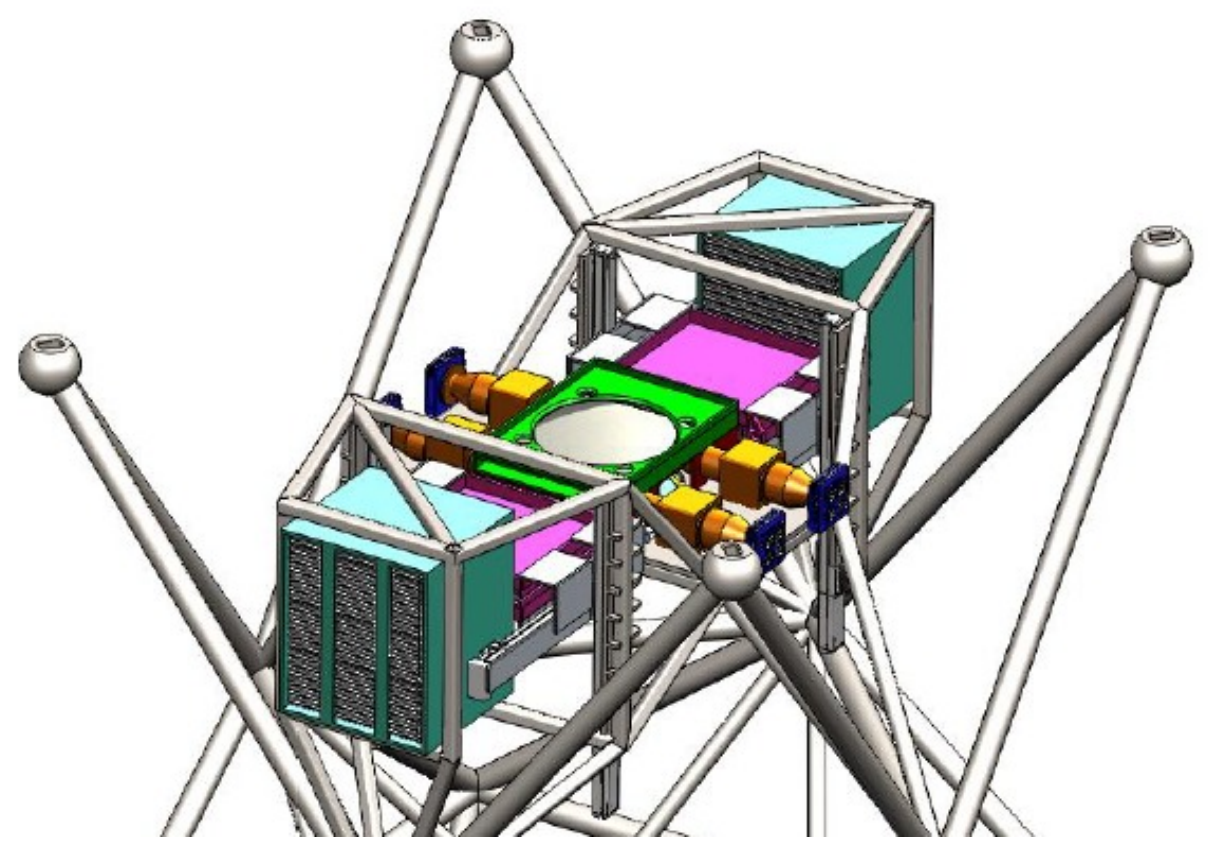

Figure 12. Layout of GMACS slit exchange unit showing the interface location for MANIFEST (green box).

MANIFEST brings significant benefits to the GMACS stand-alone modes. It provides improvement to multiplex via efficient detector packing and spectral resolution via image-slicing, and provides access to integral field unit modes. For the baseline design, two sets of fibre probes are envisioned. The first intended for survey type work, and in particular 
Lyman Alpha tomography of the intergalactic medium, comprises a set of $\sim 100,19$-fibre, 1.25" diameter field science or sky Starbugs feeding the GMACS slit. The probes are parked on a quasi-regular grid in the 20' diameter MANIFEST field. The second mode consists of a single $\sim 2100$ fibre integral field unit with a 6" diameter field. This non-moving probe is positioned near the MANIFEST field center. A slit unit would be used to select the operating mode. For MANIFEST operation, the GMACS spectrograph is located in one of the GIR bays in its stowed position. MANIFEST is deployed to the centre focal plane location. An arm holding the GMACS fibre slit is then deployed from the MANIFEST instrument structure into the GMACS slit exchange unit (see Figure 12).

The GMACS team is now in the process of refining the concept for the GMACS instrument and considering trades between field of view, wavelength coverage, resolution, and operating modes. During the MANIFEST CDS phase, the MANIFEST team will also trade off number and configuration of Starbug probes to provide the optimum science return to the GMACS instrument. The details of the opto-mechanical interfaces will also be developed.

\section{ADDITIONAL MODES}

\subsection{Wavefront sensing Starbugs}

The ability to position multiple miniaturised wavefront sensors precisely over a large focal surface is advantageous to understand the localized wavefront distortions through the atmosphere and telescope. The AAO has prototyped a compact and lightweight Shack-Hartmann wavefront-sensor that fits into a standard Starbug robot [11]. Each sensor makes use of a polymer fibre bundle to relay an image produced by a microlens array placed at the telescope focal plane in the Starbug to a re-imaging camera mounted elsewhere. The use of polymer fibre bundle allows for a high fill-factor, high throughput, low weight system in which multiple sensors can be multiplexed to a single low-noise camera. Such a sensing system opens up the possibility to perform multi-object adaptive optics. Further work is ongoing to develop miniature correcting modules that can be located underneath the field plate to provide correction for multiple objects simultaneously across the telescope field of view.

\subsection{Spectro-polarimetry}

Polarimetry can provide unique information about stellar and galactic sources that cannot be obtained with spectroscopy alone. The addition of a polarimetric capability to an astronomical multi-object spectrograph can be achieved via placement of polarizing optics in the focal plane for a slit-mask spectrograph or via employing dual fibre feeds for a fibre fed spectrograph. MANIFEST offers the potential to incorporate a multi-object spectro-polarimetric capability via deploying polarizing feed optics across the field plate (either above or below). By targeting multiple single objects, the polarizing optics are kept compact (and hence cost effective). Further effort is warranted to develop this concept in terms of the polarizing modules and their deployment mechanisms.

\subsection{Dedicated fibre spectrographs}

Fibre-fed spectrographs offer key advantages when image slicing is employed because the design of the spectrograph can be de-coupled from the design of the telescope feeding it. This means that designs can be readily exchanged between telescopes. Hector, for example, is a multi-IFU spectrograph system currently being designed for the AAT [20]. The Hector instrument has a baseline requirement for a spectrograph with a $100 \mu \mathrm{m}$ slit width (fibre core diameter) at $\mathrm{f} / 3.2$ which is equivalent to $1.6^{\prime \prime}$ sampling at the $3.9 \mathrm{~m}$ diameter AAT. This is very close to the nominal MANIFEST GMACS fibres of $90 \mu \mathrm{m}$ slit width at $\mathrm{f} / 3.3$, or $0.22^{\prime \prime}$ sampling on the $24.5 \mathrm{~m}$ GMT. The wavelength coverage (370-1000 nm) and spectral resolution $(\mathrm{R}=5000-7000)$ for Hector are both appropriate for many science cases on the GMT. Hector spectrograph designs thus may offer an opportunity for upgrading the GMT instrument suite capability in the future in a very modular way. In the next phase for MANIFEST we will further explore these synergies between MANIFEST and the Hector spectrographs.

\section{SUMMARY}

The Prototyping Design Study for MANIFEST, nearing completion, has resulted in an engineered fibre positioning system based on the technology of Starbugs parallel positioning robots to be developed to the point which it can be employed on sky to conduct the TAIPAN stellar and galaxy surveys using the UKST. Lessons learnt, as well as actual designs will be directly relevant for use in the Conceptual Design Study phase for MANIFEST that will commence shortly. The MANIFEST team will also continue with the development of the key instrument interfaces for MANIFEST to G-CLEF and GMACS, and the interfaces with GMT. 


\section{REFERENCES}

[1] Saunders, W., Colless, M., Saunders, I., Hopkins, A., Goodwin, M., Heijmans, J., Brzeski, J., and Farrell, T., "MANIFEST: a many-instrument fiber-positioning system for GMT," Proc. SPIE 7735, 773568 (2010).

[2] Goodwin, M., Brzeski, J., Case, S., Colless, M., Farrell, T., Gers, L., Gilbert, J., Heijmans, J., Hopkins, A., Lawrence, J., Miziarski, S., Monnet, G., Muller, R., Saunders, W., Smith, G., Tims, J., and Waller, L., "MANIFEST instrument concept and related technologies," Proc. SPIE 8446, 84467I (2012).

[3] Lawrence, J. S., Brown, D. M., Brzeski, J., et al., "The MANIFEST fibre positioning system for the Giant Magellan Telescope," Proc. SPIE 9147, 914794 (2014).

[4] McGrath, A., and Moore, A., "Starbug: enabling the smart focal plane," Proc. SPIE 5495, 600-610 (2004).

[5] Haynes, R., McGrath, A., Brzeski, J., Correll, D., Frost, G., Gillingham, P., Miziarski, S., Muller, R., and Smedley, S., "It's alive! Performance and control of prototype Starbug actuators," Proc. SPIE 6273, 62731V (2006).

[6] McGrath, A., and Haynes, R., "Deployable payloads with Starbug," Proc. SPIE 6273, 62731W (2006).

[7] Goodwin, M., Heijmans, J., Saunders, I., Brzeski, J., Saunders, W., Mueller, R., Haynes, R., and Gilbert, J., "Starbugs: focal plane fiber positioning technology," Proc. SPIE 7739, 77391E (2010).

[8] Gilbert, J., Goodwin, M., Heijmans, J., Mueller, R., Miziarski, S, Brzeski, J., Waller, L., Saunders, W., Bennet, A., and Tims, J., "Starbugs: all-singing, all-dancing fibre positioning robots," Proc. SPIE 8450, 84501A (2012).

[9] Brown, D,. Case, S., Gilbert, J., Goodwin, M., Jacobs, D., Kuehn, K., Lawrence, J., Lorente, N., Nichani, V., Saunders, W., Staszak, N., and Tims, J., "Starbug fibre positioning robots: performance and reliability enhancements," Proc SPIE 9151, 914710 (2014).

[10] Goodwin, M., Lorente, N. P. F., Hong, S. E., Satorre, C., and Lawrence, J., "Field target allocation and routing algorithms for starbugs," Proc. SPIE 9152, 91520S (2014).

[11] Goodwin, M., Richards, S., Zheng, J., Lawrence, J., Leon-Saval, S., Argyros, A., and Alcalde, B., "Miniaturized Shack-Hartmann wavefront sensors for starbugs," Proc. SPIE 9151, 91514T (2014).

[12] Szentgyorgyi, A., Frebel, A., Furesz, G., et al., "The GMT-CfA, Carnegie, Catolica, Chicago Large Earth Finder (G-CLEF): A General Purpose Optical Echelle Spectrograph for the GMT with Precision Radial Velocity Capability," Proc. SPIE 8446, 84461H (2012).

[13] Szentgyorgyi, A., Barnes, S., Bean, J., et al., "A preliminary design for the GMT-Consortium Large Earth Finder (G-CLEF)," Proc. SPIE 9147, 914726 (2014).

[14]DePoy, D. L., Allen, R., Barkhouser, R., et al., "GMACS: a wide field, multi-object, moderate-resolution, optical spectrograph for the Giant Magellan Telescope," SPIE Proc 8446, 84461N (2012).

[15] Kuehn, K., Lawrence, J. S., Brown, D., Case, S., Colless, M., Content, R., Gers, L., Goodwin, M., Hopkins, A., Ireland, M., Lorente, N., Muller, R., Nichani, V., Rakman, A., Saunders, W., Staszak, N., Tims, J., Waller, L., "TAIPAN: Optical Spectroscopy with StarBugs," Proc. SPIE 9147, 914710 (2014).

[16] Staszak, N., F., Lawrence, J., Brown, D. M., et al., "TAIPAN Instrument Fibre Positioner and Starbug Robots: Engineering Overview," Proc. SPIE 9912, in press (2016).

[17] Staszak, N. F., Lawrence, J., Zhelem, R., Content, R., Churilov, V., Case, S., Brown, R., Hopkins, A. M., Kuehn, K., Pai, N., Klauser, U., Nichani, V., and Waller, L., "TAIPAN Fibre Feed and Spectrograph: Engineering Overview," Proc. SPIE 9912, in press (2016).

[18]D'Odorico, V., Viel, M., Saitta, F., Cristiani, S., Bianchi, S., Boyle, B., Lopez, S., Maza, J., and Outram, P., "Tomography of the intergalactic medium with Lya forests in close QSO pairs," Mon. Not. R. Astron. Soc. 372, 1333 (2006).

[19] Croom, S. M., Lawrence, J. S., Bland-Hawthorn, J., et al., "The Sydney-AAO Multi-object Integral field spectrograph," MNRAS 421, 872 (2012). 
[20] Lawrence, J., Bland-Hawthorn, J., Bryant, J., Brzeski, J., Colless, M., Croom, S., Gers, L., Gilbert, J., Gillingham, P., Goodwin, M., Heijmans, J., Horton, A., Ireland, M., Miziarski, S., Saunders, W., Smith, G., "Hector: a high-multiplex survey instrument for spatially resolved galaxy spectroscopy," Proc SPIE 8446, 844653 (2012).

[21]De Silva, G. M., Freeman, K. C., Bland-Hawthorn, J., Asplund, M., and Bessell, M. S., "Chemically Tagging the HR 1614 Moving Group," Astron. J. 133, 694 (2007).

[22] De Silva, G. M., Freeman, K. C., Bland-Hawthorn, J., et al. "The GALAH survey: scientific motivation," Mon. Not. R. Astron. Soc. 449, 2604 (2015).

[23] Ellis, S., Sheinis, A. I., Zhelem, R., Brown, D. M., Lidman, C., Nataf, D., Casey, A., Xavier, P., Tims, J., Staszak, N. F., Lawrence, J. S., Bryant, J. J., Sharp, R., and Gillingham, P., "ULTIMATE: a deployable multiple integral field unit for Subaru," Proc. SPIE 9908, in press (2016).

[24] GMT Organisation, "GMT System Level Preliminary Design Review - Telescope (Section 6)”, GMTO, 18 Dec 2013, <http://www.gmto.org/slpdr/> (15 June 2016).

[25] Saunders, W., Gillingham, P., Smith, G., Kent, S., Doel, P., "Prime focus wide-field corrector designs with lossless atmospheric dispersion correction," Proc. SPIE 9151, 91511M (2016).

[26] Nariai, K., and Takeshi, K. K., "Primary corrector for SUBARU Telescope with a new type of atmospheric dispersion corrector," Proc. SPIE 9151, 230 (2014).

[27] Sheinis, A., Borja, A., Asplund, M., et al, "First light results from the High Efficiency and Resolution MultiElement Spectrograph at the Anglo-Australian Telescope," J. Astron. Tele. Instr. Sys. 1, 035002 (2015).

[28] Ellis, S. C., Ireland, M., Lawrence, J. S., et al., "KOALA: a wide-field 1000 element integral-field unit for the Anglo-Australian Telescope," Proc. SPIE 8446, 84460V (2012).

[29] DESI Collaboration, "DESI Final Design Report Part II: Instrument Design”, DESI Collaboration, 1 March 2014, <http://desi.lbl.gov/wp-content/uploads/2014/04/fdr-instrument-biblatex2.pdf> (6 June 2016).

[30]Lorente, N. P. F., Vuong, M. V., Hong, S. E., Goodwin, M., Kuehn, K., Satorre, C., Farrel, T., and Shortridge, K., "AAO Starbugs: software control and path-finding algorithms," Proc. SPIE 9913, in press (2016).

[31]Farrell, T., and Shortridge, K., "DRAMA2 - DRAMA for the modern era," Proc. ICALEPCS'15, in press (2015). 